\title{
The Use of Wavelet Transform To Evaluate The Sensitivity of Acoustic Emission Attributes To Variation of Cutting Parameters in Milling Aluminium Alloys
}

Reza Asadi

Iran University of Science and Technology

Mohamad Javad Anahid

Iran University of Science and Technology

Hoda Heydamia

The University of British Columbia

Hedayeh Mehmanparast

University of Concordia

Seyed Ali Niknam ( $\sim$ seyed-ali.niknam@polymtl.ca )

Polytechnique Montreal

Research Article

Keywords: Acoustic Emission, Signal Processing, Wavelet Transform, Milling, Aluminum 7075

Posted Date: November 15th, 2021

DOI: https://doi.org/10.21203/rs.3.rs-1054589/v1

License: (c) (1) This work is licensed under a Creative Commons Attribution 4.0 International License. Read Full License 


\section{Abstract}

Appropriate acquisition and assessment of the dominant acoustic emission (AE) signal attributes generated under various experimental cutting conditions may provide significant knowledge. Consequently, it enhances the efficiency in manufacturing process monitoring and control. However, according to the literature, a lack of information was noticed on the behavior of $A E$ signal attributes under various cutting conditions. Considering that milling is among the most widely used machining operations, the aim of this investigation is to acquire adequate knowledge about interactions between cutting parameters and their direct and indirect effects on the obtained AE signals attributes from the milling process. In the course of this work, the effects of cutting conditions on the attributes calculated from wavelet transform (WT) of AE signals will be presented. WT signal processing was conducted with five models of mother wavelets, and appropriate decomposition numbers were deployed. The approximated signal attributes obtained from each decomposition were assessed. According to signal processing and statistical calculations, cutting speed, feed rate, and coating significantly impacted the variation of AE signal attributes. Also, the most sensitive AE signal attributes and decompositions were rms, std, entropy and energy, and 2nd and 6th decompositions, respectively. The outcome of this work can be integrated into advanced artificial intelligence (Al) approaches to implement real-time monitoring of manufacturing processes.

\section{Introduction}

Milling is among the most widely used machining processes, which includes a sophisticated morphology and encompasses complicated interactions between cutting parameters, workpiece, and cutting tools [1]. In recent decades, aluminum alloys have found widespread applications in numerous industrial sectors and products due to their specific characteristics, including a considerable strength-to-weight ratio, high conductivity, and relatively good machinability[2, 3].

Nowadays, advanced methods and strategies, including Al algorithms were widely used to analyze sensory signals (e.g., AE signals) and improve the efficiency of machining process monitoring $[4,5]$. The term AE refers to transient elastic waves obtained from the rapid release of energy from one or more sources when the material is under stress [6]. In recent decades, extensive research has been conducted on the use of $A E$ in various applications. $A E$ also offers significant advantages over other NDT approaches due to its unique ability to monitor manufacturing systems such as power generation, healthcare, offshore and onshore structures, and damage pattern recognition $[7,8]$. One of the primary applications of sound signals or $\mathrm{AE}$ signals in the machining industry is tool condition monitoring [4]. It was assessed whether such signals could be used to monitor and detect critical machining attributes, such as surface roughness, surface quality, tool wear, tool failure, chip thickness, chatter vibration, tool life, etc. [5, 9-17]. The majority of recent research projects in AE signal usage in machining have focused on Al-based control methods [18-20]. Tool condition monitoring and tool wear measurement using AE signals information are more efficient than ambient signals and vibrations due to the broader range of frequencies covered by $\mathrm{AE}$ sensors [5,21].

Knowing their impressive adaption abilities to acquire more satisfying results, researchers have adopted $A E$ signals along with the required sensors and modelling methods in a vast number of recent research in the field of machining and specifically high precision processes [22-24]. Murakami, et al. [22] tried to detect the contact of the cutting tool and workpiece within the micromachining process by the usage of AE signals and built-in AE sensors. Zanger, et al. [24] used FEM simulation and AE signals obtained from piezoelectric sensors for the cutting operation of Ti-Al-4V to evaluate the influence of cutting parameters and analysis of segmentation frequency of such operations.

Reliable analysis based on AE signals requires parallel consideration of the physical and mechanical conditions of the experiments and understanding the signal behavior. In machining operations, AE signals' behavior depends on several parameters, such as cutting parameters, tools, and workpiece properties. Besides, the AE attributes in different domains must be well identified and confirmed to enhance the performance and accuracy of the monitoring process [12, 13]. Analyzing the AE signal can be investigated in the time, frequency, and time-frequency (wavelet transform) domain. Various research works on signal processing of the extracted AE signals in the milling process were reported in $[25,26]$. The main achievements of existing research works are automatic detection [27], surface roughness prediction [28], and modeling slot milling process [26]. Additional studies, including tool wear monitoring in end milling of aluminum-ceramic composites [5] and surface integrity analysis for high-speed machining in the frequency domain [29], were conducted using AE signals attributes. The use of AE signal attributes in the time-frequency domain was also investigated [30].

Despite the significant applications of AE signals in condition monitoring and health diagnosis of machining processes, a lack of knowledge in the timefrequency domain sensitivity of these signals to various parameters involved in the machining process is evident. This deficiency is more pronounced in milling processes where the significant effects of interaction, complex chip formation modes and pressure in different directions are their main characteristics. There is a substantial need to further study the effects of different cutting conditions on AE signals and their features in the time-frequency domain to resolve the knowledge gap mentioned previously. The aim of this work, as aforementioned, is to assess the sensitivity of the attributes of the wavelet transform of $\mathrm{AE}$ signals to various cutting conditions in milling aluminum alloy 7075-T6. Towards this end, an advanced novel method was deployed based on the proposed method's wavelet transformations and statistical analysis. The second section comprises the theoretical background of AE signals. The experimental plan was provided in the third section. The research methodology was comprehensively discussed in section four. Section five consists of the results of the proposed methods and a discussion of them. Finally, the last part introduces a thorough conclusion of this research.

\section{Acoustic Emission Signal}

\subsection{DEFINITION AND SOURCES OF AE}

In metal machining operations, AE signals are mainly a source of information on plastic deformation and crack generation [31]. The main requirements in this area include AE receivers and sensors, AE signals, AE waveguides, machine tools, background noise, and coupling. Mechanical energy, which is the consequence of releasing elastic waves with a frequency range of $100-1000 \mathrm{kHz}$, leads to AE signals generation. Generally, piezoelectric sensors are adopted 
to detect and convert the generated waves into electrical signals. However, as shown in Figure 1, each AE event caused by energy dissipation lasts for a very short period (milliseconds range).

\subsection{SOURCES OF AE SIGNAL}

Many studies have evaluated the fundamental sources of AE in deformed/machined materials [32-35]. Based on the reported works, the underlying sources of AE signals in the metal cutting processes are attributed to (a) workpiece plastic deformation caused during the machining; (b) chip plastic deformation; (c) flank wear caused by friction between the tool flank face and the workpiece; ( $\mathrm{d}$ crater wear caused by friction between the tool rake face and chip; (e) chip breakage; (f) tool fracture and (g) collisions between chip and tool chip breakage. The acquired AE signals from milling processes containing attributes are usually continuous or transient (burst) signals [35]. Tool fracture and chip breakage are undeniable sources of transient signals (Figure 2) and can be clearly understood to be the result of processes (e) to $(\mathrm{g})$. On the other hand, continuous signals result from deformation and wear on the rake/flank sides of the cutting tools, and the mentioned items (a) to (d) are the key sources of this signal.

\subsection{AE PARAMETERS}

When processing the wavelet transform of AE signals, several attributes can be derived and assessed. Table 1 lists the extracted wavelet transform decomposition-based AE signal attributes. In Appendix I the formulas of such parameters are provided.

Table 1

The extracted wavelet features from AE signals

\begin{tabular}{|ll|}
\hline List & AE parameters \\
\hline 1 & Maximum: max \\
\hline 2 & Minimum: min \\
3 & Root Mean Square: rms \\
\hline 4 & Standard deviation: std \\
\hline 5 & Energy: enrg \\
\hline 6 & Entropy: entrpy \\
\hline 7 & Kurtosis: kur \\
\hline 8 & Skewness: skew \\
\hline 9 & Crest Factor: crest \\
\hline 10 & Impulse Factor: impulse \\
\hline 11 & 4 th Moment: mom4 \\
\hline 12 & FM4 \\
\hline
\end{tabular}

\section{Experimental Procedures}

\subsection{Experimental plan}

A multilevel full factorial design was constructed in this work to draw the experimental plan. The experimental conditions and their levels were presented in Table 2. The experiments were conducted on a three-axis CNC machine (power: 50kW, speed: $28000 \mathrm{rpm}$; torque: $50 \mathrm{Nm}$ ) with the details and levels introduced in Table 2. It is noteworthy that a total of $54\left(3^{3} \times 2^{1}\right)$ tests were performed on AA7075-T6 alloy with three types of coated tools. To ensure the quality stability of the experimental conditions, preliminary tests were performed, including the stability of cutting processes, tool vibration, and system dynamic behavior. In addition, an AE sensor was installed two meters away from the chip formation zone to investigate the background noise level. The result indicates a high signal-to-noise ratio (SNR), indicating that the background noise has negligible effects on the signals obtained in the vicinity of the chip formation zone (the first sensor).

Furthermore, after observing a slight deflection in the workpiece and the cutting tool during the conducted preliminary tests to guarantee the stability of the process and prevent possible tool wear from affecting the AE signals, new inserts were deployed after each machining test. Tables 3-4 provide the list of parameters and attributes of the workpiece and cutting tools. The following section presents the method for this process, including the signal processing method and data analysis. 
Table 2

Cutting parameters and their levels used

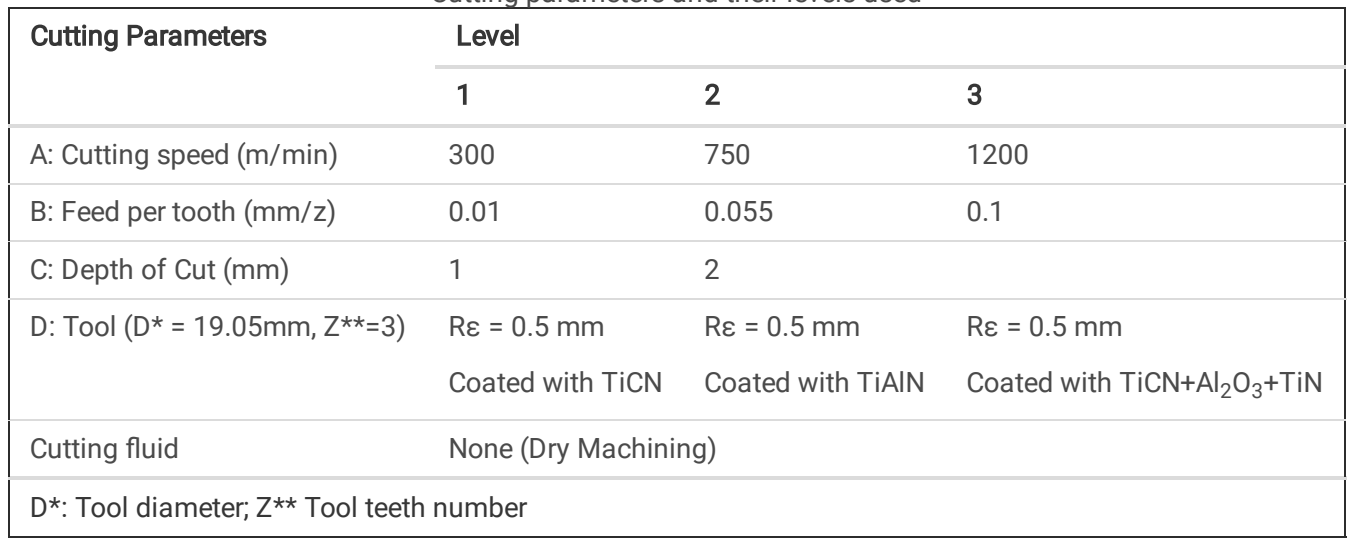

Table 3

Physical characteristics of the material used [36]

\begin{tabular}{|ll|}
\hline Physical parameters & Materials \\
\cline { 2 - 2 } & AA 7075 - T6 \\
\hline Brinell Hardness (HB) & 150 \\
\hline Elongation (\%) & 11 \\
\hline Elastic limit (MPa) & 503 \\
\hline Mechanical resistance (MPa) & 572 \\
\hline
\end{tabular}

Table 4

Parameters of the cutting tools used [37]

\begin{tabular}{|llll|}
\hline Operational conditions & \multicolumn{4}{l|}{ Cutting tool (Iscar Ref: E90A-D.75-W.75-M) } \\
\hline Coating & TiCN & TiAIN & TiCN+Al ${ }_{2} \mathrm{O}_{3}+\mathrm{TiN}$ \\
\hline Insert nose radius $\mathrm{R} \varepsilon[\mathrm{mm}]$ & $\mathrm{R} \varepsilon=0.5$ & $\mathrm{R} \varepsilon=0.83$ & $\mathrm{R} \varepsilon=0.5$ \\
Reference insert & IC 328 & IC 908 & IC 4050 \\
\hline
\end{tabular}

\section{$\mathrm{AE}$ signal monitoring system}

Figures 3 and 4 show the AE data acquisition system utilized in this study, including two AE TEDS microphones, a data processing unit, and how the system is set up. Figure 3 a shows a comprehensive signal processing unit. Microphones 1(ref:2564023) and 2(ref:2564024) in Figures 3b and 3c were installed near the chip formation zone and at a distance of two meters from it, respectively. The first microphone was used to obtain AE signals and the second to measure background noise. The arrangement of the workpieces used during the machining process is shown in Figure $3 \mathrm{~d}$. The schematic view of the experiments is shown in Figure 4. After implementing the proposed setup, the microphones are calibrated with a signal of $10,000 \pm 100 \mathrm{~Hz}$ to ensure their efficiency and reliability.

\section{Research methodology}

Figure 5 shows the required strategy to complete the studies to investigate the effects of cutting parameters on the features of the original AE signals obtained from 0.2 seconds of cutting operation. The steps of this strategy are as follows:

Installation of $\mathrm{AE}$ sensors $\left(f_{s}=100 \mathrm{KHz}\right)$ near the chip formation zone for data acquisition and also Kistler-9255B three-axis dynamometer $\left(f_{s}=48 \mathrm{kHz}\right)$ to determine the cutting forces in the coordinate axes.

1. Implementation of milling tests with the proposed conditions and parameters on AA7075-T6 alloy.

2. Obtaining AE time-domain signals $(x(t))$ based on specified cutting parameters such as cutting speed, cutting depth, feed per tooth, coated materials (Figure 4b).

3. Conversion of time-domain AE signals $(\mathrm{x}(\mathrm{t}))$ into time-frequency domain data based on the wavelet transform analysis $\left(D W T_{\psi}^{x}\right)$ and process of obtained $A E$ signals in the time-frequency domain.

4. Optimal features extraction is based on different decompositions (from details and approximated AE signals) and utilization of different mother wavelets. 5. Perform statistical analysis to determine the attributes of the processed AE signals with the highest sensitivity to the cutting parameters used in each experiment. 
6. Machining aluminum alloys involves difficulties such as burr formation, BUE, and wear modes, including adhesion [38-41]. Therefore, to minimize the risk of noted challenges and also to avoid the possible negative impacts on the recorded results, the following assumptions are considered:

1. During the implementation of the preliminary experimental tests that were performed to validate test conditions, the machining process was proved to be stable. It is noteworthy that cutting tools and fixtures were observed without any deflection. Additionally, there was no chatter vibration detected in the tests.

2. New inserts were used at each stage of the designed experiments to avoid the risk of deviations in the test outcomes and enhance the milling process measurements' accuracy.

The statistical analysis introduced significant parameters, models, decompositions, and mother wavelets by criteria such as $\mathrm{P}$-value, $\mathrm{R}^{2}$ and $\mathrm{R}^{2}$ adj $($ section 4.1).

\subsection{Method of analysis}

according to the detailed introduction of statistical terms used to analyze data in [42], the following experimental techniques and criteria have been used to identify effective machining parameters on the values of features extracted from wavelet transform signal processing:

1. ANOVA: The analysis of variance (ANOVA) assesses any significant relationship between cutting parameters and obtained AE signal attributes at $95 \%$ confidence interval (Cl).

- In statistics principles, the coefficient of determination $\left(\mathrm{R}^{2}\right)$ indicates the measure of the variability of a dependent variable based on the independent variable(s). Here, this criterion indicates the sensitivity of an extracted AE feature to variation of cutting parameters. Whereas $\mathrm{R} 2>0.85$ indicates significant sensitivity to variation of experimental parameters, $\mathrm{R} 2<0.85$ shows that the extracted attributes are considered non-sensitive to such parameters.

- $\mathrm{R}^{2}$ adj is a modified version of $\mathrm{R}^{2}$ and can provide a more detailed view of that correlation. This criterion is equivalent or smaller than $\mathrm{R}^{2}$.

- $P$-value: Determines whether your experimental test results are statistically significant or not. This study adopted this criterion to confirm the significance of the cutting parameters and the proposed model based on the linear, second-order degree or 2-factor interactions. The following evaluation policy was utilized:

- P-value < 0.05; denotes the proposed model/parameter is significant

- P-value $>0.1$; denotes the proposed model/parameter is insignificant

- $0.05<$ P-value $<0.1$; proves that the proposed model/parameter is mid-significant

2. Pareto chart: provides a visual demonstration of statistical analysis of proposed experimental cutting parameters (individual and their combined effects) on the extracted AE signal attributes employing decreasing contribution.

Statistical criteria, including P-value, $\mathrm{R}^{2}$, and $\mathrm{R}^{2}$ adj, were used to determine the significant and insignificant parameters. It is to say that the models presented in this work are second-order degree models. The $A E$ signal responses with an $\mathrm{R}^{2}$ less than 0.85 were also insensitive to various cutting parameters as inputs. Also, with magnitudes, less than 0.05 as P-value, the cutting parameters and their interactions were considered statistically influential parameters.

\section{Results And Discussion}

After processing the measured AE signals and implementing the strategies for suitable selection of features as described in Figure 5, an assessment of the sensitivity of the experimental cutting parameters to AE signal attributes was conducted. The measured AE signals were decomposed through six decompositions by five mother wavelets, so-called haar, db2, db10, sym8, and bior1.3. After selecting decomposed signals' attributes, a thorough assessment was performed by statistical examinations of the AE attributes. Figure 6 shows maximum $\mathrm{R}^{2}$ values for the most sensitive signal attributes belonging to the three most significant mother wavelets for approximate and detailed signal types. Based on Figure 6, the signal attribute "max" (for all three mother wavelets) has the highest $R^{2}$ values among all five signal attributes within approximate signals and the lowest for detail signals, having the same $R^{2}$ value of about $80 \%$ for both approximate and detail signals. It means that the "max" attribute does not have a wide difference for approximate and detail signals. All four other signal attributes, including rms, std, energy and entropy, have roughly the same $\mathrm{R}^{2}$ values for each of approximate and detail signals. However, the mentioned four attributes for detail signals have $\mathrm{R}^{2}$ values $20 \%$ more than approximate signals. In other words, the sensitivity of rms, std, energy, and entropy is $20 \%$ higher for detail signals. Therefore, regardless of the mother wavelet type, they were preferred over approximate signals for detecting the changes in machining operations when cutting parameters change. It should be noticed that for attribute "max" the second type of mother wavelet (db2) had a better performance. Due to page limit and long discussion needed, other examined AE signal attributes were not presented in Figure 6.

Figure 7 summarizes the $\mathrm{R}^{2}$ values of signal attributes, including rms, std, energy and entropy for all decompositions and all five mother wavelets studied. Different decompositions do not have the same capability to present sensitive features related to their various details and characteristics. Considering Figure 7, generally, it can be seen that decompositions 2 and 6 offer higher $\mathrm{R}^{2}$ values as compared to other decompositions. Thus, it can be easily understood that rms, std, energy, and entropy in the second and sixth decompositions of detail signals have acceptable sensitivity according to the introduced criteria. Besides, Figure 6 and Figure 7 show that $\mathrm{R}^{2}$ values obtained from the detail signals using the bior1.3, haar, and db2 mother wavelets had the best results, respectively. For further clarification, Table 6 presents the $\mathrm{R}^{2}$ and $\mathrm{R}^{2}$ adj values of the rms, std, energy, and entropy attributes for decompositions 2 and 6 , and bior 1.3 , haar, and $\mathrm{db} 2$ mother wavelets for the detail signals. Except for minor cases where their $\mathrm{R}^{2}$ was less than acceptable, the bior 1.3 , haar, and db2 mother wavelets in decompositions 2 and 6 provided sensitive attributes with acceptable accuracy.

Table 5. $\mathrm{R}^{2}$ and $\mathrm{R}^{2}$ adj values of the rms, std, energy, and entropy features for decompositions 2 and 6 , using the bior1.3, haar, and db2 mother wavelets 
Table 5

$R^{2}$ and $R^{2}$ adj values of the rms, std, energy, and entropy features for decompositions 2 and 6 , using the bior1.3, haar, and db2 mother wav

\begin{tabular}{|c|c|c|c|c|c|c|c|c|c|c|c|c|c|c|}
\hline \multirow{3}{*}{$\begin{array}{l}R^{2} \& \\
R^{2}{ }_{\text {adj }}\end{array}$} & \multicolumn{8}{|c|}{ 2nd Decomposition } & \multicolumn{6}{|c|}{ 6th Decomposition } \\
\hline & \multicolumn{2}{|l|}{$\mathrm{ms}$} & \multicolumn{2}{|l|}{ std } & \multicolumn{2}{|l|}{ energy } & \multicolumn{2}{|l|}{ entropy } & \multicolumn{2}{|l|}{ rms } & \multicolumn{2}{|l|}{ std } & \multicolumn{2}{|l|}{ energy } \\
\hline & $\mathrm{R}^{2}$ & $\mathbf{R}^{2}$ adj & $\mathrm{R}^{2}$ & $\mathbf{R}^{2}$ adj & $\mathbf{R}^{2}$ & $\mathrm{R}^{2}{ }_{\mathrm{adj}}$ & $\mathbf{R}^{2}$ & $\mathbf{R}^{2}$ adj & $\mathbf{R}^{2}$ & $\mathrm{R}^{2}{ }_{\mathrm{adj}}$ & $\mathbf{R}^{2}$ & $\mathbf{R}^{2}$ adj & $\mathbf{R}^{2}$ & $\mathbf{R}^{2}$ adj \\
\hline Bior1.3 & 91.459 & 88.198 & 91.697 & 88.998 & 89.525 & 86.121 & 88.894 & 85.285 & 89.579 & 85.942 & 88.8799 & 85.2659 & 86.3223 & 81.877 \\
\hline Haar & 91.77 & 88.45 & 91.33 & 88.51 & 88.91 & 85.31 & 89.91 & 86.61 & 89.85 & 86.32 & 88.84 & 85.22 & 86.25 & 81.78 \\
\hline $\mathrm{Db} 2$ & 90.94 & 87.99 & 90.45 & 87.86 & 88.57 & 84.86 & 89.92 & 86.65 & 85.32 & 81.24 & 86.53 & 82.152 & $82.53^{*}$ & $76.85^{\star}$ \\
\hline
\end{tabular}

Table 6

$\mathrm{R}^{2}$ and $\mathrm{R}^{2}$ adj values of the most significant proposed features using 5 different mother Wavelets on accumulated detailed signals

\begin{tabular}{|c|c|c|c|c|c|c|c|c|c|c|}
\hline \multirow{2}{*}{$R^{2} \& R^{2}{ }_{a d j}$} & \multicolumn{2}{|l|}{ Haar } & \multicolumn{2}{|l|}{ Db2 } & \multicolumn{2}{|l|}{ Db10 } & \multicolumn{2}{|l|}{ Sym8 } & \multicolumn{2}{|c|}{ Bior1.3 } \\
\hline & $\mathbf{R}^{2}$ & $\mathrm{R}^{2}$ adj & $\mathrm{R}^{2}$ & $\mathbf{R}_{\text {adj }}$ & $\mathbf{R}^{2}$ & $\mathbf{R}^{2}$ adj & $\mathbf{R}^{2}$ & $\mathbf{R}_{\text {adj }}$ & $\mathrm{R}^{2}$ & $\mathbf{R}^{2}{ }_{\text {adj }}$ \\
\hline $\max$ & 82.27 & 76.51 & 84.45 & 79.4 & 81.09 & 78.9 & 83.61 & 79.25 & 81.58 & 75.6 \\
\hline rms & 75.87 & 68.03 & 78.22 & 71.14 & 81.08 & 74.93 & 80.87 & 74.66 & 75.45 & 67.48 \\
\hline std & 75.87 & 68.03 & 78.22 & 71.14 & 81.08 & 74.93 & 80.87 & 74.66 & 75.45 & 67.48 \\
\hline energy & 66.98 & 56.25 & 69.03 & 58.96 & 70.99 & 61.56 & 70.87 & 61.4 & 66.57 & 55.7 \\
\hline entropy & 76.31 & 68.61 & 78.48 & 71.49 & 81.64 & 75.67 & 81.56 & 75.57 & 77.1 & 69.66 \\
\hline
\end{tabular}

Table 6 shows $\mathrm{R}^{2}$ and $\mathrm{R}^{2}$ adj values for the accumulated detailed signals. It can be observed that the signal resulting from the sum of the detail signals of all decompositions do not provide significant performance. The accumulated detail signal is not separated into different decompositions and consequently includes additional details from the machining process. As a result, it adversely affects the performance of sensitivity criteria. Figure 8 illustrates the Pareto charts of rms, std, energy, and entropy (features with highest values of $\mathrm{R}^{2}$ ) obtained from the most appropriate mother wavelets. Based on the presented charts, rms, std, and energy were substantially controlled with the variation of all the proposed cutting parameters, including the cutting speed (A), feed per tooth (B), coating material (D), and depth of cut (C) respectively while for entropy depth of cut (C) can not be assumed a significant parameter. There is a correlation between the levels of proposed cutting parameters, including cutting speed, feed per tooth, coating strength and depth of cut (not being effective on entropy level) and rms, std, energy, and entropy, with $\mathrm{V}_{\mathrm{c}}$ as the most effects factor on all the mentioned attributes. The obtained analysis revealed that a considerable proportion of AE signals generated during milling processes are closely linked to levels of consumed energy and material removal rate (MRR), as stated in [43]. Other significant factors on rms and std are the interaction between cutting speeds (AA), coating materials (DD), feed per tooth and depth of cut $(B C)$. The most visible inputs affecting the entropy are cutting speed and feed per tooth (AB). However, in comparison to rms and std, the energy was affected by more statistically significant parameters, including coupled interactions between cutting speeds (AA), coating materials (DD), feed per tooth and depth of cut $(B C)$, cutting speed and feed per tooth ( $A B)$, cutting speed and depth of cut ( $A C)$, and cutting speed and coating materials (AD).

In the frequency domain, the conclusions revealed that peak amplitude and peak frequency entitled as the most sensitive factors to input catting parameters. They were not still satisfactorily governed by such parameters [44]. The negligible P-Value $(<<0.05)$ of feed per tooth, cutting speed, coating material, and depth of cut when taking rms, std, energy and entropy throughout the wavelet transform analysis of decomposed AE detail signals of milling of Aluminium 7075 was observed and considered. It approves that these cutting parameters remarkably control the variation in maximum amplitude. Cutting speed, depth of cut, coating material details, and feed per tooth shows a strong correlation with AE signals in the time-frequency domain, confirming that AE signals and cutting parameters can be selected appropriately for the purpose of monitoring machining processes. The order of influential cutting parameters on significant attributes of AE signals was shown in Table 7. It is noteworthy that, in general, the milling process signals are more sophisticated than other non-traditional machining methods. As a result, they are significantly affected by system deviations. These signals can include background noise such as mechanical, electrical, or acoustic. Aluminum alloys machining presents challenges such as burr formation, BUE, and adhesion of work parts to the cutting tools [38-41].

The present study certifies the accuracy and effectiveness of AE signal information in milling processes monitoring. According to the obtained information, it can be claimed that AE signals change more by the variation of cutting parameters (cutting speed and feed per tooth) than by changing the coatings. In this study, a second-order model is used to investigate the sensitivity of AE signal parameters, and as the subject of further studies, it is suggested to evaluate more models for statistical analysis on machining data. Furthermore, the method introduced in this study can be utilized with Al-based techniques (e.g., Neural/Deep Network) to develop a robust classification and predictive model and monitor machining operations [45]. Besides, for non-deflecting signals, higher frequency ranges, advanced filtering, and anti-aliasing algorithms are recommended. Finally, it should be noted that the theory of predicting the AE signal parameters is an acceptable approach to avoid the need for repeated tests. 
Table 7

Effective cutting attributes on sensitive AE parameters

\begin{tabular}{|lllll|}
\hline AE Parameters & Cutting speed & Feed per tooth & Depth of cut & Coating material \\
\hline rms & 1 & 2 & 4 & 3 \\
\hline std & 1 & 2 & 4 & 3 \\
\hline energy & 1 & 2 & 3 & 4 \\
entropy & 1 & 2 & $4^{*}$ & 3 \\
\hline
\end{tabular}

$\left.{ }^{*}\right)$ indicates Non-statically effective factors

\section{Conclusion}

By conducting experimental machining tests (milling) on AA 7075 workparts and implementing proposed AE signal decomposition in time-frequency domain (wavelet transform), feature extraction and statistical analysis, the sensitivity of extracted AE signal attributes was examined with different cutting parameters. In addition, the sensitivity of AE signal attributes to variation of cutting parameters were also evaluated. This study would illuminate and cover the lack of knowledge about the impact of cutting parameters on the significant AE signal attributes to enhance the online monitoring machining process.

These conclusions could be drawn from the findings presented in the article:

1. Rms, std, energy, and entropy were the most sensitive AE signal attributes to variation of cutting conditions.

2. In decompositions \#2 and \#6, sensitive AE attributes had higher $\mathrm{R}^{2}$ values than in other decompositions. It was also noticed that detail signals were more efficient in detecting the variation of cutting conditions and coating material properties compared to approximate signals.

3. Cutting speed, feed per tooth, coating, and depth of cut (except in the case of entropy) were the most influential factors on rms, std, energy, and entropy. Other significant factors were interaction effects between cutting parameters, with different intensities of influence on each attribute. The mentioned parameters' coupled interaction was the most influential cutting conditions on rms, std, and energy. However, the energy was also influenced by the coupled interaction between cutting speed and feed per tooth, cutting speed and depth of cut, and cutting speed and coating material. Entropy was influenced only by coupled interaction between cutting speed and feed per tooth.

4. As a result of this study, it was demonstrated that $A E$ signals could be used for monitoring milling processes using wavelet domains. Consequently, $A E$ signal information can be used in milling processes, which was previously denied due to noise, friction, chip accumulation, and lack of accurate extraction and selection of sensory signal attributes.

5. Compared with time and frequency analyses, wavelet transform led to a better determination of sensitive signal features with more accuracy and confidence. Therefore, it is recommended to use the wavelet transform for signal processing when dealing with high interaction environments such as machining operations.

6. A combination of Al-based methods, high quality and precise multiple sensors, a higher frequency range of data, and advanced filtering and anti-aliasing algorithms to develop the efficiency of the proposed algorithm is suggested.

7. A comprehensive and precise presentation of significant cutting conditions and sensitive $A E$ signal features to variation input cutting parameters undoubtedly facilitates the predictive fault models and online monitoring systems for diverse machining processes.

\section{Declarations}

\section{Acknowledgment}

This work was financially supported by the Natural Sciences and Engineering Research Council of Canada (NSERC) and Fonds de recherche du Québec Nature et technologies (FRQNT).

Funding: Not applicable

Conflicts of Interest: The authors declare no conflict of interest. The funders had no role in the design of the study, in the collection, analyses, or interpretation of data, in the writing of the manuscript, or in the decision to publish the results.

Availability of data and material: The authors confirm that the data supporting the findings of this study are available within the article [and/or] its supplementary materials.

Code availability: Not applicable

Ethics approval: Not applicable

Consent to participate: Not applicable

Consent for publication: All authors permit the publisher to publish the work.

Author Contributions: The research results in this work were presented in the B.Sc thesis of Mr. Anahid. Mr. Asadi, Ms. Heydarnia, and Dr. Mehmanparast helped him in the broader scope of experimental works and data analysis. They also paid special effort to manuscript preparation. Dr. Niknam also acted as 
the supervisor of Mr. Anahid. All authors have read and agreed to the published version of the manuscript.

\section{References}

1. R. Asadi, A. Yeganefar, and S. A. Niknam, "Optimization and prediction of surface quality and cutting forces in the milling of aluminum alloys using ANFIS and interval type 2 neuro fuzzy network coupled with population-based meta-heuristic learning methods," The International Journal of Advanced Manufacturing Technology, vol. 105, pp. 2271-2287, 2019.

2. T. Sugihara, Y. Nishimoto, and T. Enomoto, "On-machine tool resharpening process for dry machining of aluminum alloys employing LME phenomenon," Precision Engineering, vol. 40, pp. 241-248, 2015.

3. J. Guo, J. Zhang, H. Wang, K. Liu, and A. S. Kumar, "Surface quality characterisation of diamond cut V-groove structures made of rapidly solidified aluminium RSA-905," Precision Engineering, vol. 53, pp. 120-133, 2018.

4. M. C. Gomes, L. C. Brito, M. B. da Silva, and M. A. V. Duarte, "Tool wear monitoring in micromilling using Support Vector Machine with vibration and sound sensors," Precision Engineering, vol. 67, pp. 137-151, 2021.

5. P. Twardowski, M. Tabaszewski, M. Wiciak-Pikuła, and A. Felusiak-Czyryca, "Identification of tool wear using acoustic emission signal and machine learning methods," Precision Engineering, 2021.

6. S. A. Niknam, V. Songmene, and Y. J. Au, "Proposing a new acoustic emission parameter for bearing condition monitoring in rotating machines," Transactions of the Canadian Society for Mechanical Engineering, vol. 37, pp. 1105-1114, 2013.

7. J. Zhu, B. He, X. Wang, L. Cui, and L. Yuhao, "Extraction of partial discharge signal feature based on dual-tree complex wavelet transform and singularvalue decomposition," in 2018 Condition monitoring and diagnosis (CMD), 2018, pp. 1-5.

8. J. Wei, H. Wang, B. Lin, T. Sui, F. Zhao, and S. Fang, "Acoustic emission signal of fiber-reinforced composite grinding: frequency components and damage pattern recognition," The International Journal of Advanced Manufacturing Technology, vol. 103, pp. 1391-1401, 2019.

9. M. A. Camara, A. M. Abrao, J. C. C. Rubio, G. C. D. Godoy, and B. S. Cordeiro, "Determination of the critical undeformed chip thickness in micromilling by means of the acoustic emission signal," Precision Engineering, vol. 46, pp. 377-382, 2016.

10. S. Sun, X. Hu, and W. Zhang, "Detection of tool breakage during milling process through acoustic emission," The International Journal of Advanced Manufacturing Technology, 2020/07/16 2020.

11. R. Pahuja and M. Ramulu, "Surface quality monitoring in abrasive water jet machining of Ti6Al4V-CFRP stacks through wavelet packet analysis of acoustic emission signals," The International Journal of Advanced Manufacturing Technology, vol. 104, pp. 4091-4104, 2019/10/01 2019.

12. C. XiaoQi, Z. Hao, and D. Wildermuth, "In-process tool monitoring through acoustic emission sensing," Automated Material Processing Group, Automation Technology Division, vol. 1, 2001.

13. X. Chen and B. Li, "Acoustic emission method for tool condition monitoring based on wavelet analysis," The International Journal of Advanced Manufacturing Technology, vol. 33, pp. 968-976, 2007.

14. N. Mokhtar, I. Y. Ismail, M. Asmelash, H. Zohari, and A. Azhari, "Analysis of acoustic emission on surface roughness during end milling," ARPN Journal of Engineering and Applied Sciences, vol. 12, pp. 1324-1328, 2017.

15. Z. Li, G. Wang, and G. He, "Surface quality monitoring based on time-frequency features of acoustic emission signals in end milling Inconel-718," The International Journal of Advanced Manufacturing Technology, vol. 96, pp. 2725-2733, 2018.

16. P. Sutowski, M. Sutowska, and W. Kapłonek, "The use of high-frequency acoustic emission analysis for in-process assessment of the surface quality of aluminium alloy 5251 in abrasive waterjet machining," Proceedings of the Institution of Mechanical Engineers, Part B: Journal of Engineering Manufacture, vol. 232, pp. 2547-2565, 2018.

17. M. Dib, J. Duduch, and R. Jasinevicius, "Minimum chip thickness determination by means of cutting force signal in micro endmilling," Precision Engineering, vol. 51, pp. 244-262, 2018.

18. M. A. Hasan, M.-H. Abu-Bakar, R. Razuwan, and Z. Nazri, "Deep Neural Network Tool Chatter Model for Aluminum Surface Milling Using Acoustic Emmision Sensor," in MATEC Web of Conferences, 2018, p. 03003.

19. P. Krishnakumar, K. Rameshkumar, and K. Ramachandran, "Machine learning based tool condition classification using acoustic emission and vibration data in high speed milling process using wavelet features," Intelligent Decision Technologies, vol. 12, pp. 265-282, 2018.

20. M. Elforjani and S. Shanbr, "Prognosis of bearing acoustic emission signals using supervised machine learning," IEEE Transactions on industrial electronics, vol. 65, pp. 5864-5871, 2017.

21. J. V. Abellan-Nebot and F. R. Subirón, "A review of machining monitoring systems based on artificial intelligence process models," The International Journal of Advanced Manufacturing Technology, vol. 47, pp. 237-257, 2010.

22. H. Murakami, A. Katsuki, T. Sajima, K. Uchiyama, K. Houda, and Y. Sugihara, "Spindle with built-in acoustic emission sensor to realize contact detection," Precision Engineering, vol. 70, pp. 26-33, 2021.

23. N. Dubey, A. Roushan, U. Rao, K. Sandeep, and K. Patra, "Tool condition monitoring in micro-end milling using wavelets," in IOP Conference Series: Materials Science and Engineering, 2018, p. 012045.

24. F. Zanger, A. Kacaras, M. Bächle, M. Schwabe, F. P. León, and V. Schulze, "FEM simulation and acoustic emission based characterization of chip segmentation frequency in machining of Ti-6Al-4V," Procedia CIRP, vol. 72, pp. 1421-1426, 2018.

25. T. Mohanraj, J. Yerchuru, H. Krishnan, R. N. Aravind, and R. Yameni, "Development of tool condition monitoring system in end milling process using wavelet features and Hoelder's exponent with machine learning algorithms," Measurement, vol. 173, p. 108671, 2021. 
26. M. Luo, J. Mei, and D. Zhang, "Time-domain modeling of a cutter exiting a workpiece in the slot milling process," Chinese Journal of Aeronautics, vol. 29, pp. 1852-1858, 2016.

27. H. Gaja and F. Liou, "Automatic detection of depth of cut during end milling operation using acoustic emission sensor," The International Journal of Advanced Manufacturing Technology, vol. 86, pp. 2913-2925, 2016.

28. S. Nouhi and M. Pour, "Prediction of surface roughness of various machining processes by a hybrid algorithm including time series analysis, wavelet transform and Multi View Embedding," Measurement, p. 109904, 2021.

29. R. Pawade and S. Joshi, "Analysis of acoustic emission signals and surface integrity in the high-speed turning of Inconel 718 ," Proceedings of the Institution of Mechanical Engineers, Part B: Journal of Engineering Manufacture, vol. 226, pp. 3-27, 2012.

30. W. Lee, M. Ratnam, and Z. Ahmad, "Detection of chipping in ceramic cutting inserts from workpiece profile during turning using fast Fourier transform (FFT) and continuous wavelet transform (CWT)," Precision Engineering, vol. 47, pp. 406-423, 2017.

31. K. Tonphong "Bearing Condition Monitoring using Acoustic Emission and Vibration," Ph.D. Thesis,Brunel University,UK, 2002.

32. S. Lee and D. Lee, "In-process monitoring of drilling burr formation using acoustic emission and a wavelet-based artificial neural network," International Journal of Production Research, vol. 46, pp. 4871-4888, 2008.

33. A. J. Mian, N. Driver, and P. T. Mativenga, "Chip formation in microscale milling and correlation with acoustic emission signal," The International Journal of Advanced Manufacturing Technology, vol. 56, pp. 63-78, 2011.

34. D.-E. Lee, I. Hwang, C. M. Valente, J. Oliveira, and D. A. Dornfeld, "Precision manufacturing process monitoring with acoustic emission," in Condition Monitoring and Control for Intelligent Manufacturing, ed: Springer, 2006, pp. 33-54.

35. E. Rubio, R. Teti, and I. Baciu, "Advanced signal processing in acoustic emission monitoring systems for machining technology," in Intelligent Production Machines and Systems, ed: Elsevier, 2006, pp. 1-6.

36. S. A. Niknam, "Burrs understanding, modeling and optimization during slot milling of aluminium alloys," École de technologie supérieure, 2013.

37. A. Tiabi, "Formation des bavures dùsinage et finition de pieces " M.Sc Thesis, , M.Sc Thesis, École de technologie superieure, Canada, 2010.

38. S. A. Niknam and V. Songmene, "Factors governing burr formation during high-speed slot milling of wrought aluminium alloys," Proceeding of the Institution of Mechanical Engineers, Part B: Journal of Engineering Manufacture, vol. 227, pp. 1165-1179, 2013.

39. S. A. Niknam and V. Songmene, "Analytical modelling of slot milling exit burr size," The International Journal of Advanced Manufacturing Technology, vol. 73, pp. 421-432, 2014.

40. S. A. Niknam and V. Songmene, "Burr formation and correlation with cutting force and acoustic emission signals," Proceedings of the Institution of Mechanical Engineers, Part B: Journal of Engineering Manufacture, p. 0954405415590562, 2015.

41. Songmene V., R. Khettabi, and J. Kouam, "High Speed Machining: A Cost Effective \& Green Process," Int. J. Manufacturing Research (IJMR), vol. 7, pp. 229-256, 2012.

42. M. S. Phadke, Quality engineering using robust design: Prentice Hall Englewood Cliffs, NJ, 1989.

43. D. Dornfeld, "Acoustic emission feedback for precision deburring," CIRP Annals-Manufacturing Technology, vol. 41, pp. 93-96, 1992.

44. M. J. Anahid, H. Heydarnia, S. A. Niknam, and H. Mehmanparast, "Evaluating the sensitivity of acoustic emission signal features to the variation of cutting parameters in milling aluminum alloys: Part A: frequency domain analysis," Proceedings of the Institution of Mechanical Engineers, Part B: Journal of Engineering Manufacture, p. 0954405420949127, 2020.

45. A. Jardine, D. Lin, and D. Banjevic, "A review on machinery diagnostics and prognostics implementing condition-based maintenance," Mechanical Systems and Signal Processing, vol. 20, pp. 1483-1510, 2006.

\section{Figures}




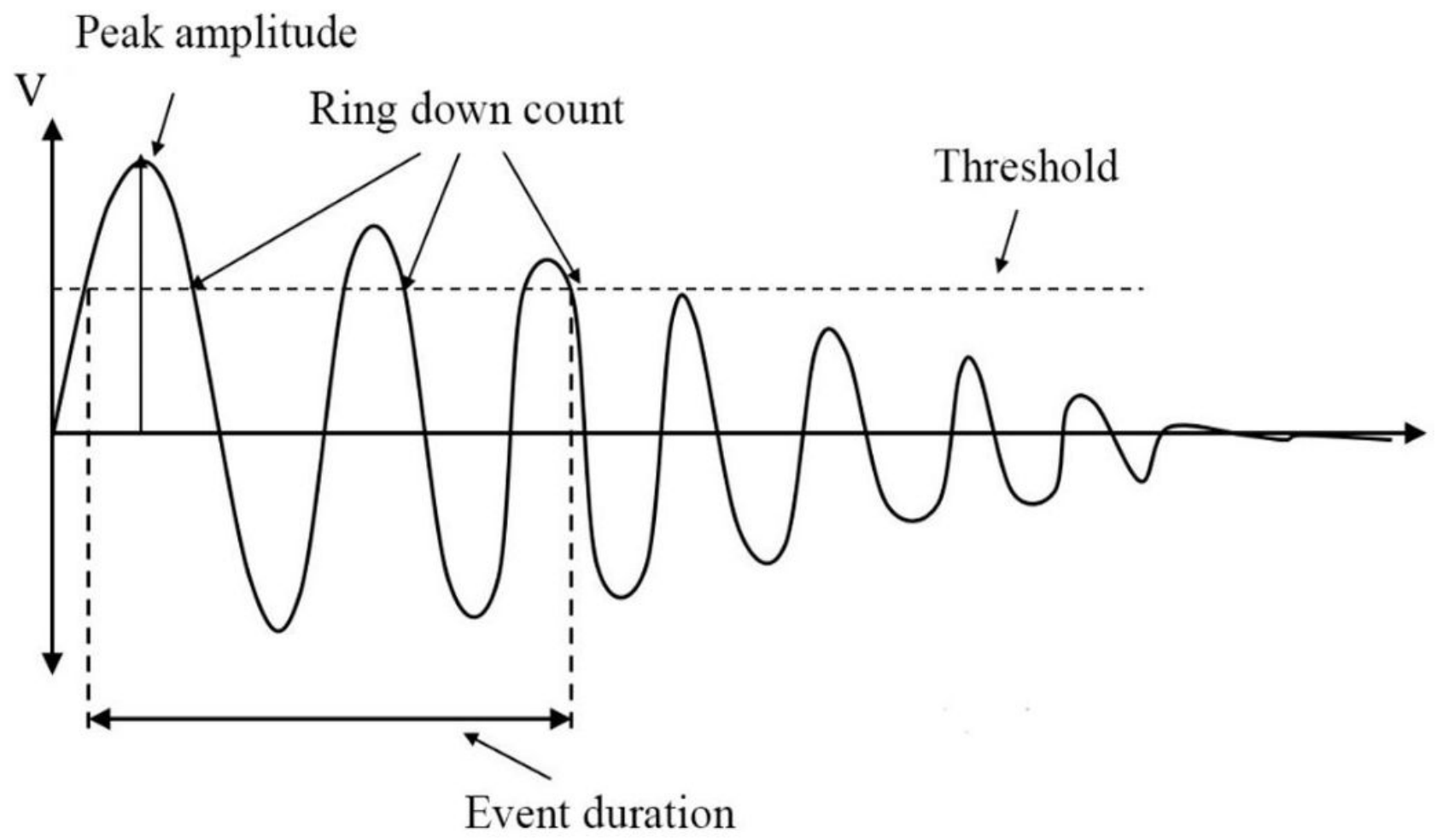

Figure 1

AE signal attributes [31]

a) Continuous AE signal Transient AE signal

b)

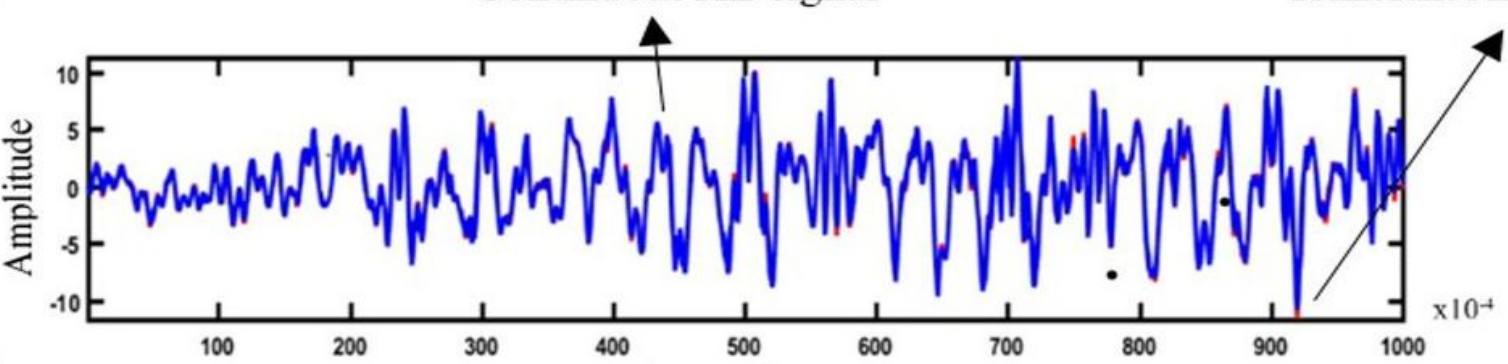

Time [ms]

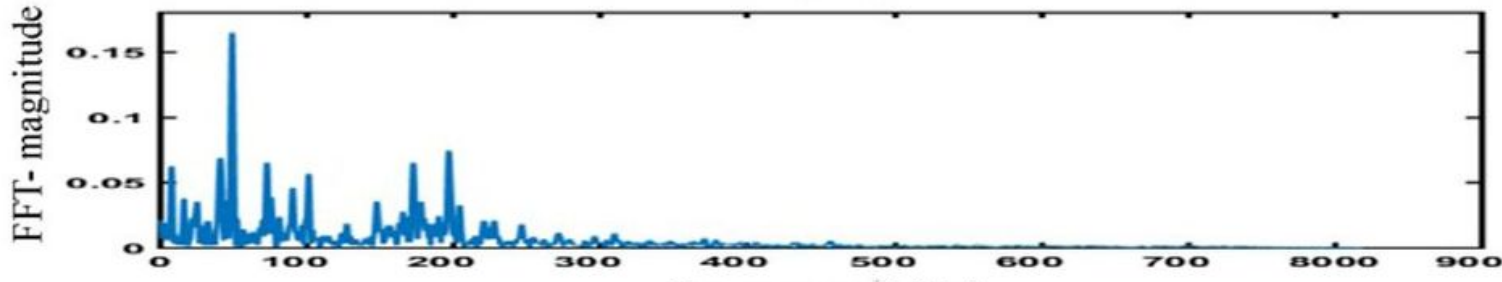

c) Frequency [kHz]

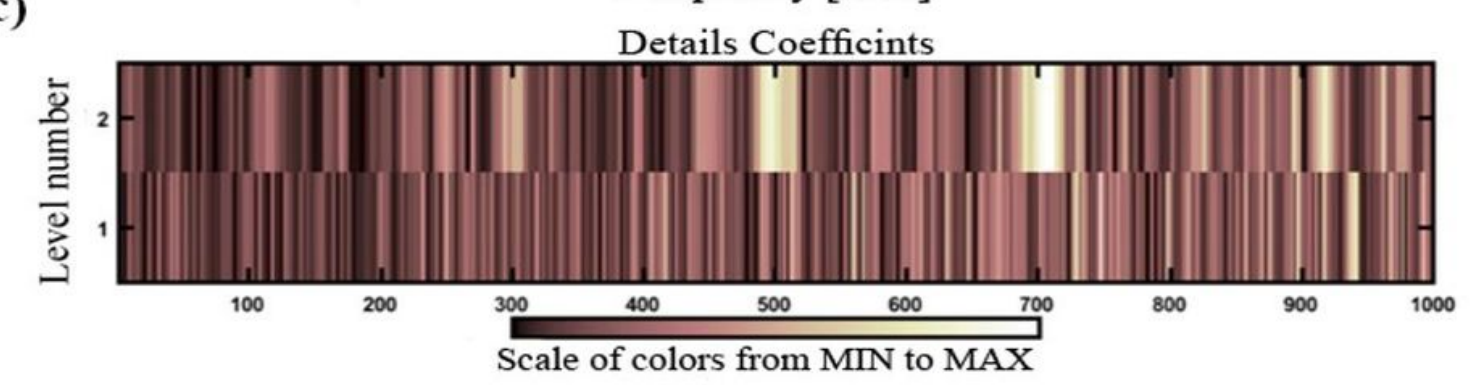


Figure 2

Samples of AE signal attributes in (a) time domain and (b) frequency domain (c) wavelet domain with two decompositions

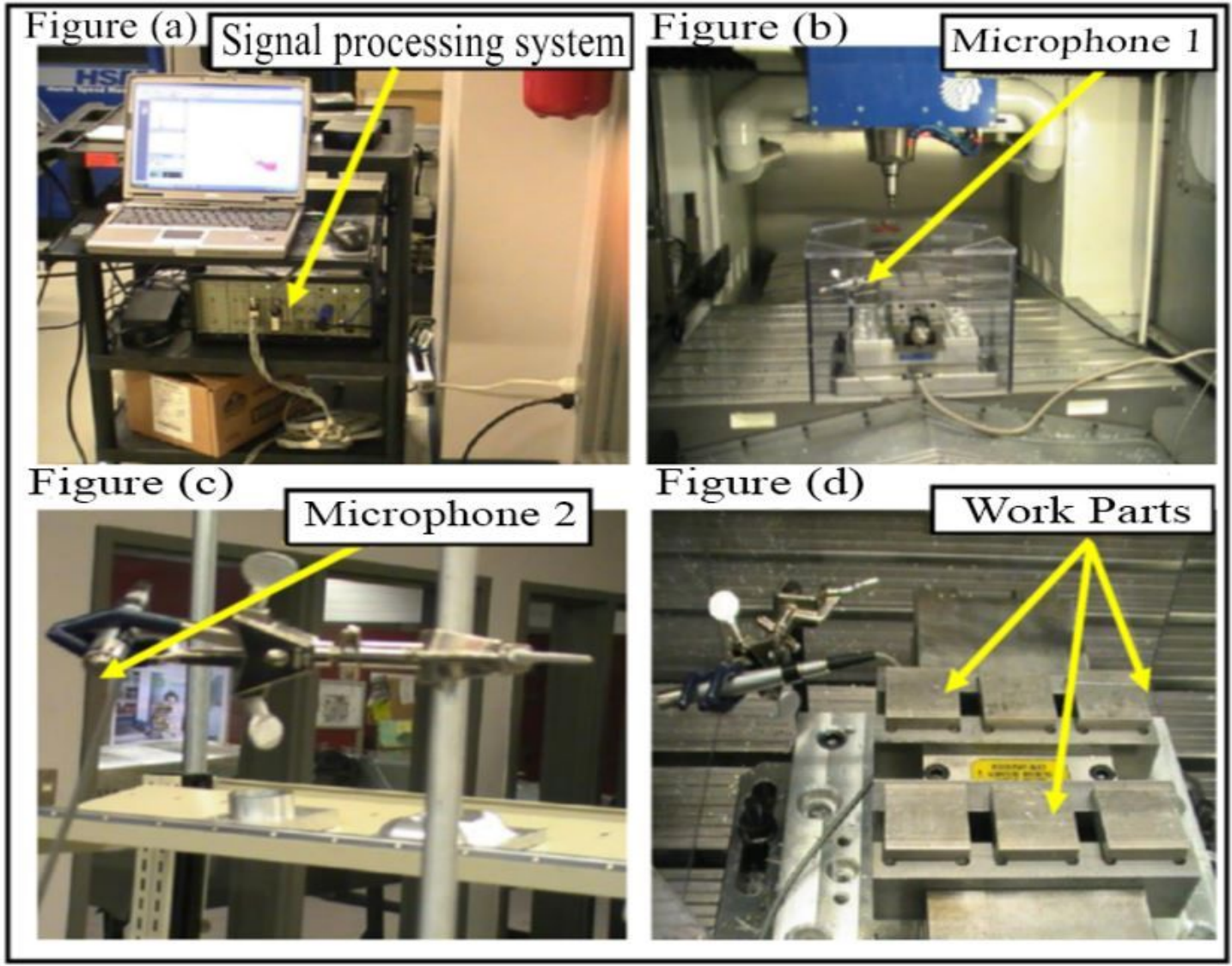

Figure 3

AE Acquisition system

(a)

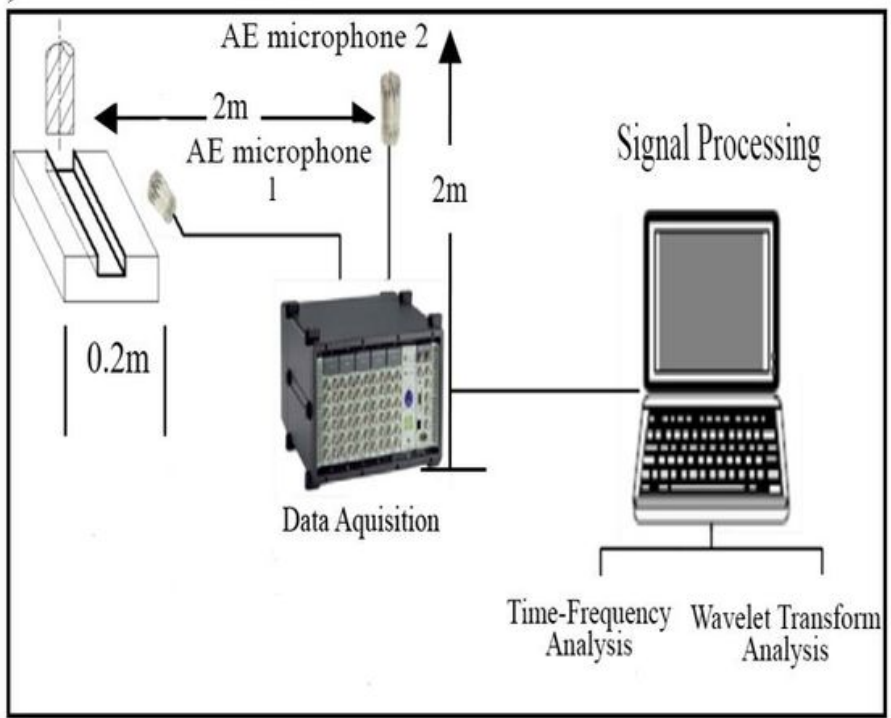

(b)

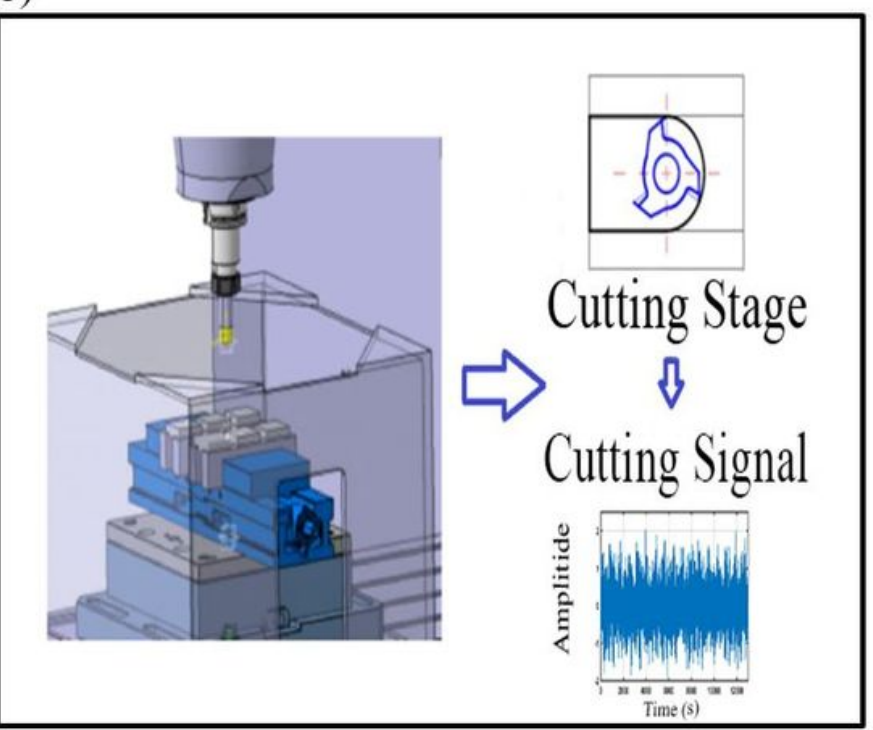


Figure 4

(a) The AE signal acquisition system used (b) Schematic overview of machining set-up and data acquisition

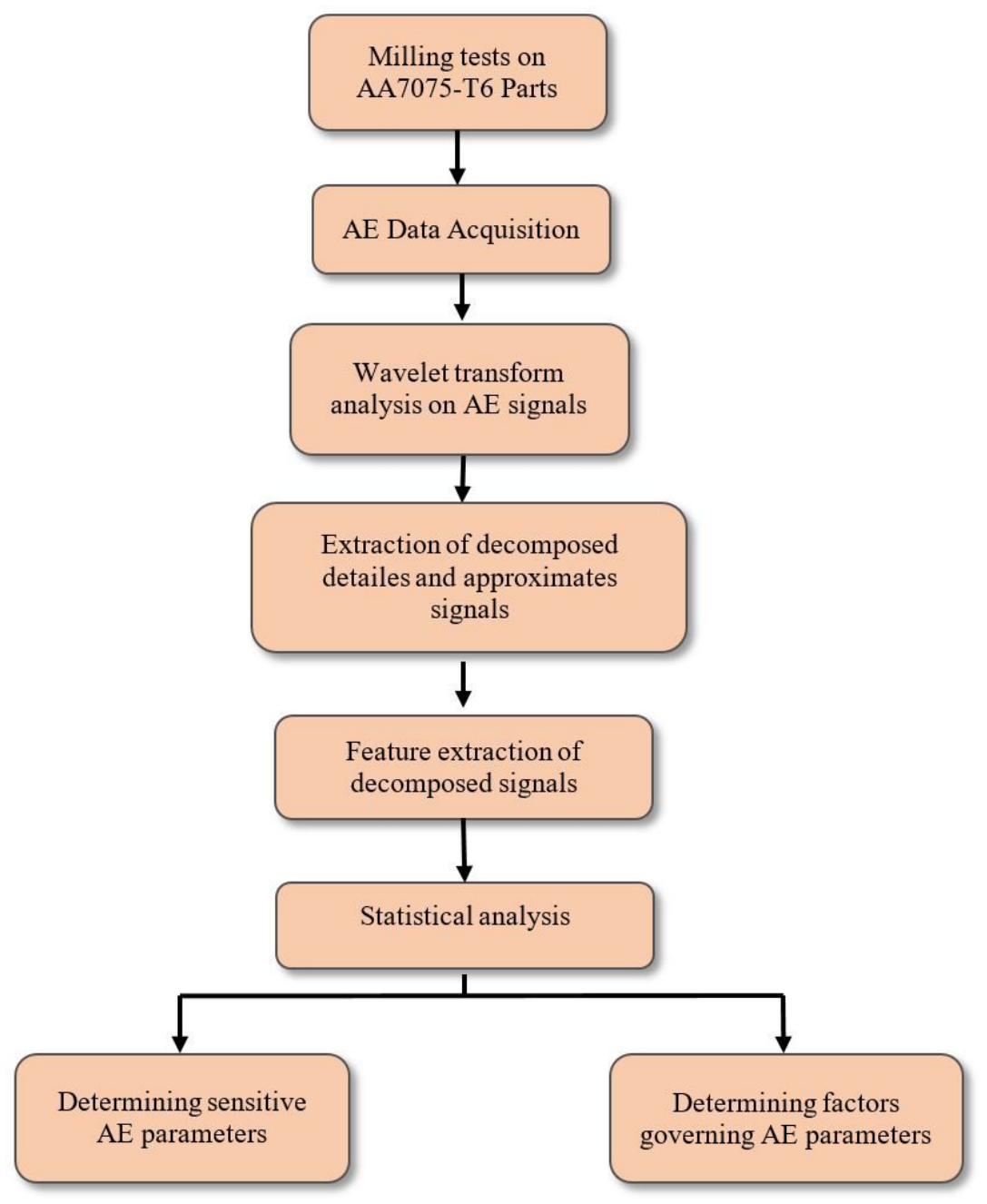

\section{Figure 5}

Scheme of the proposed methodology 

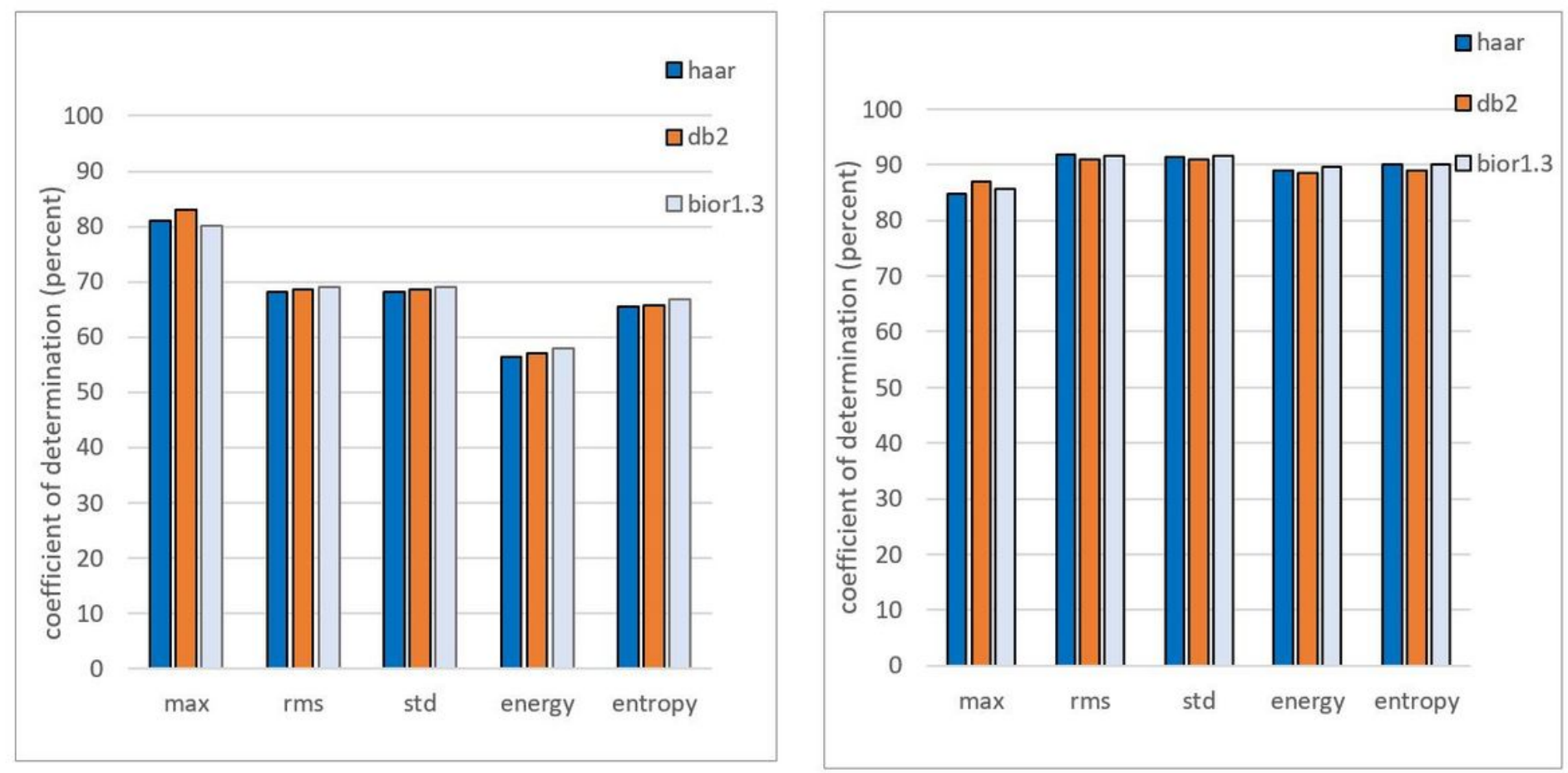

Figure 6

Coefficient of determination (R2) calculated for three main mother wavelets for different decompositions (a) approximated and (b) detailed signals
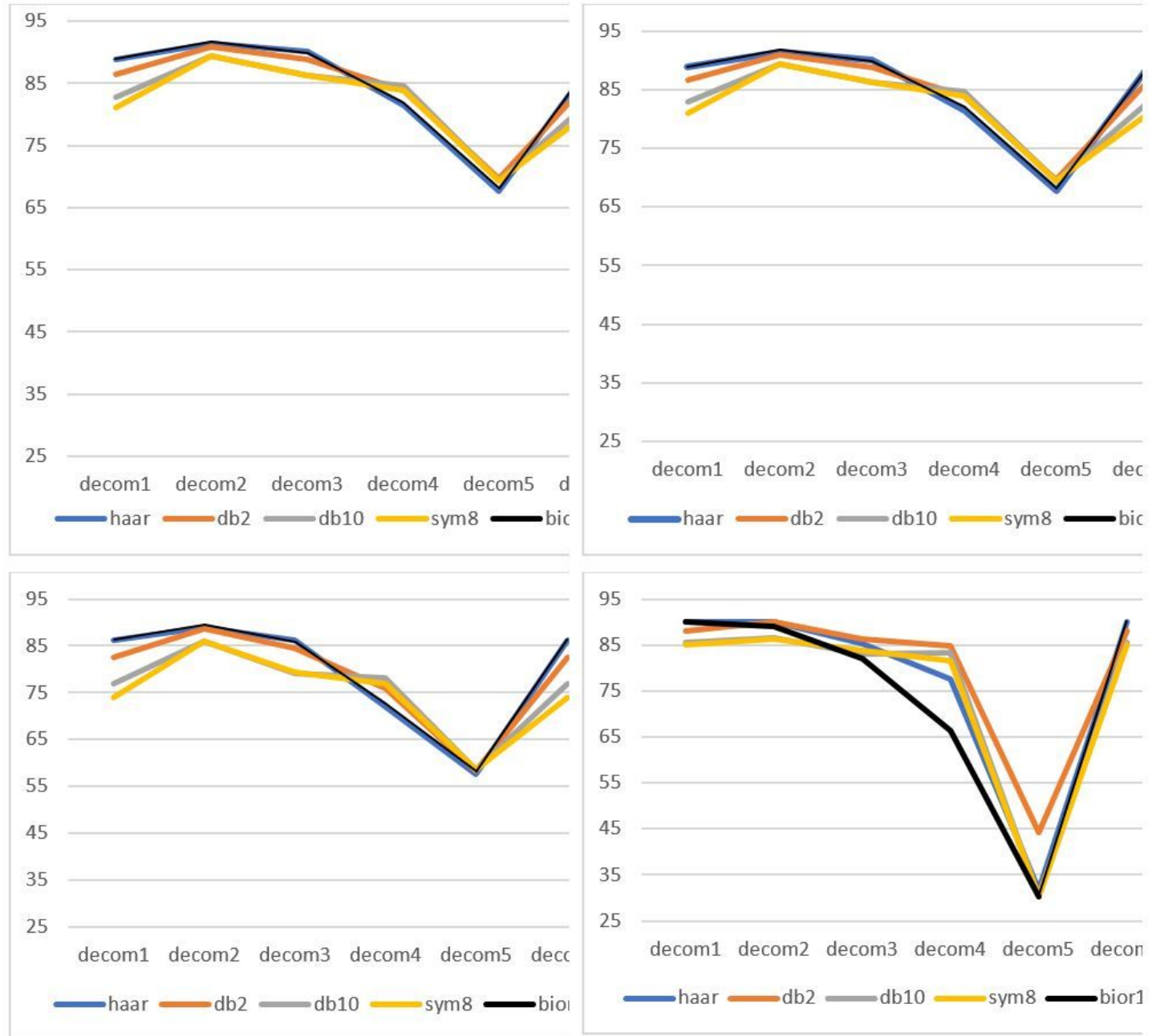

Page $13 / 14$ 
Figure 7

R2 changes based on five proposed mother Wavelets for (a)rms (b)std (c)energy, and (d) entropy as the most sensitive features
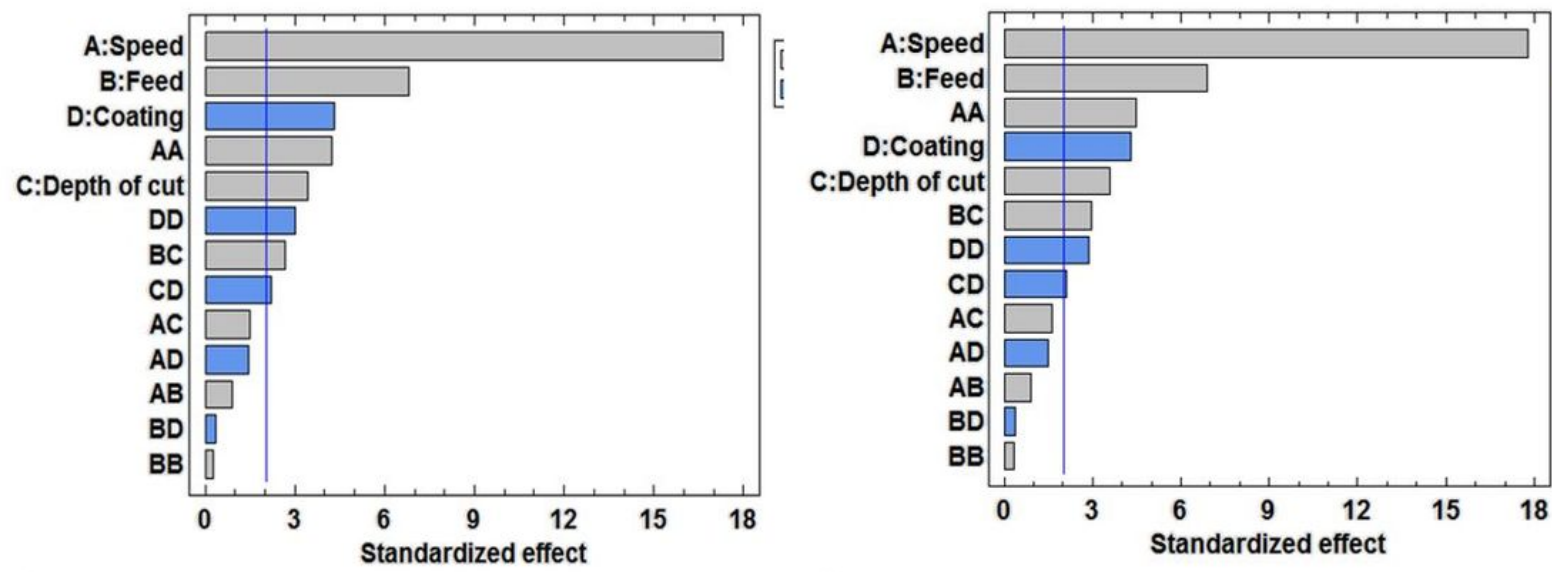

(a)

(b)
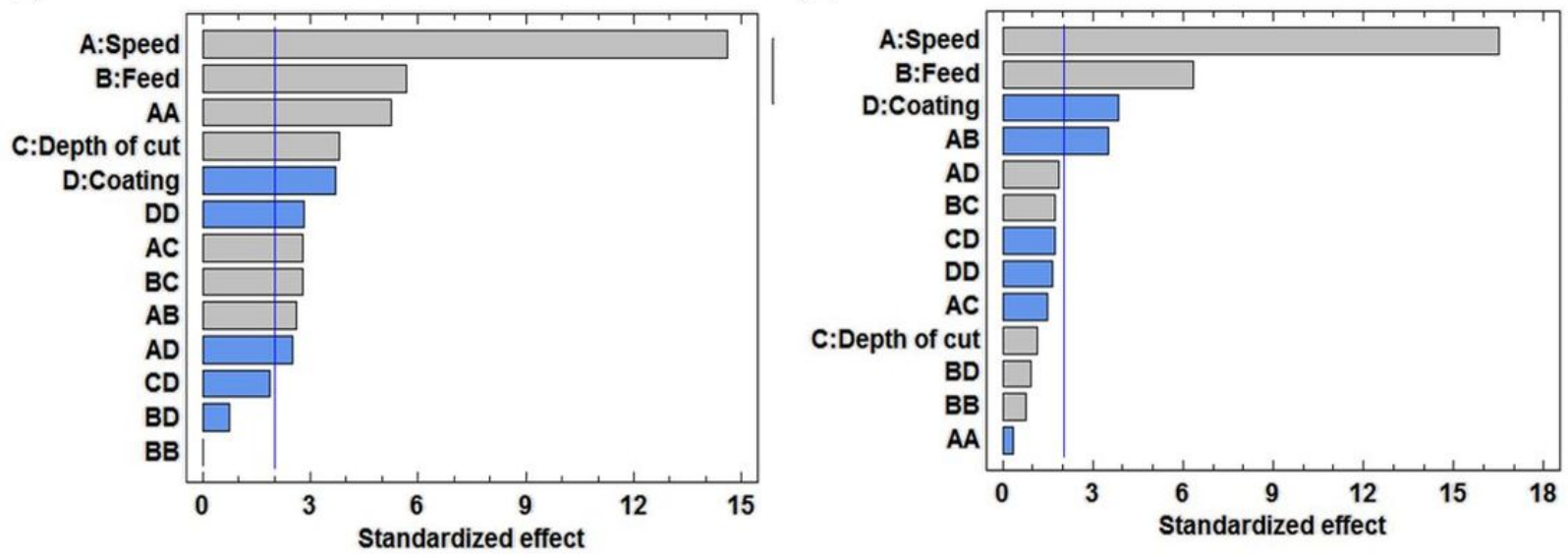

(c)

(d)

Figure 8. Pareto charts of sensitive features including (a) rms (b) std (c) energy and

Figure 8

Pareto charts of sensitive features including (a) rms (b) std (c) energy and (d) entropy with highest values of R2

\section{Supplementary Files}

This is a list of supplementary files associated with this preprint. Click to download.

- Appendix.docx 